\title{
Acinar with ductal and mucinous adenocarcinoma of prostate cancer complicated with lung metastasis: a case report and literature review
}

\author{
Yankang Cui", Chenkui Miao", Aiming Xu, Zengjun Wang, Bianjiang Liu \\ Department of Urology, The First Affiliated Hospital of Nanjing Medical University, Nanjing, China \\ \#These authors contributed equally to this work. \\ Correspondence to: Bianjiang Liu. Department of Urology, The First Affiliated Hospital of Nanjing Medical University, No. 300, Guangzhou Road, \\ Nanjing, China. Email: bjliu@njmu.edu.cn.
}

\begin{abstract}
Acinar adenocarcinoma, ductal adenocarcinoma and mucinous adenocarcinoma are the subtypes of prostate cancer (PCa). Most of the pathological types of PCa are acinar adenocarcinoma, while ductal adenocarcinoma and mucinous adenocarcinoma are uncommon. The case of acinar adenocarcinoma with ductal and mucinous adenocarcinoma has not been reported before. Herein, we report a treatment experience involving a 72-year-old man who presented similarly as most PCa patients, but the pathologic diagnosis was acinar adenocarcinoma with focal ductal and mucinous adenocarcinoma differentiating. Besides, this case is associated with lung metastasis, after radical prostatectomy (RP) and endocrine therapy the pulmonary nodule exerted a shrinking trend and the PSA level of this patient is still maintained at $0 \mathrm{ng} / \mathrm{mL}$ till now. Through literature review, we found that patients who diagnosed as mixed pathological type of PCa had a lower survivor than pure PCa patients. Furthermore, there is no corresponding consensus or guideline for treating such multiple differentiated PCa patients. Surprisingly, this patient showed a high sensitivity to androgen deprivation therapy (ADT). Although the tumor presented aggressiveness, the followup results were satisfactory and we will continue to pay attention to his physical condition. We report this case to provide a treatment strategy for the patients with multi-differentiated PCa complicated with organ metastases.
\end{abstract}

Keywords: Prostate cancer (PCa); ductal adenocarcinoma; mucinous adenocarcinoma; androgen deprivation therapy (ADT); case report

Submitted Feb 12, 2020. Accepted for publication May 26, 2020.

doi: $10.21037 /$ apm-20-386

View this article at: http://dx.doi.org/10.21037/apm-20-386

\section{Introduction}

Prostate cancer (PCa) is a major lethal malignant tumor in men of Europe and America, which has been becoming the first cancer with high ratio of incidence and mortality in the United States. PCa is the third malignant tumor in the urogenital system of the elderly after bladder and kidney tumor, and the incidence is increasing year by year (1). As ductal and mucinous adenocarcinoma are rare subtypes of PCa, there are no specific guidelines to follow in the management of these patients yet. Androgen deprivation therapy (ADT) would not be a good choice for the type of mucinous adenocarcinoma, but radical cystoprostatectomy may be a better treatment (2). After treated 19 Hong KongChinese patients with ductal adenocarcinoma of the prostate (DAP) (3), Kan et al. found a high failure rate with either RP or ADT. Furthermore, patients with pure adenocarcinoma PCa have a better clinical outcome after RP than patients with mixed ductal or mucinous adenocarcinoma $(2,4)$. In addition to the rare pathological type of this case, the pulmonary nodule was also notable. Here, we describe a 72-year-old male patient, who presented with a multidifferentiated PCa complicated by lung metastasis, which 

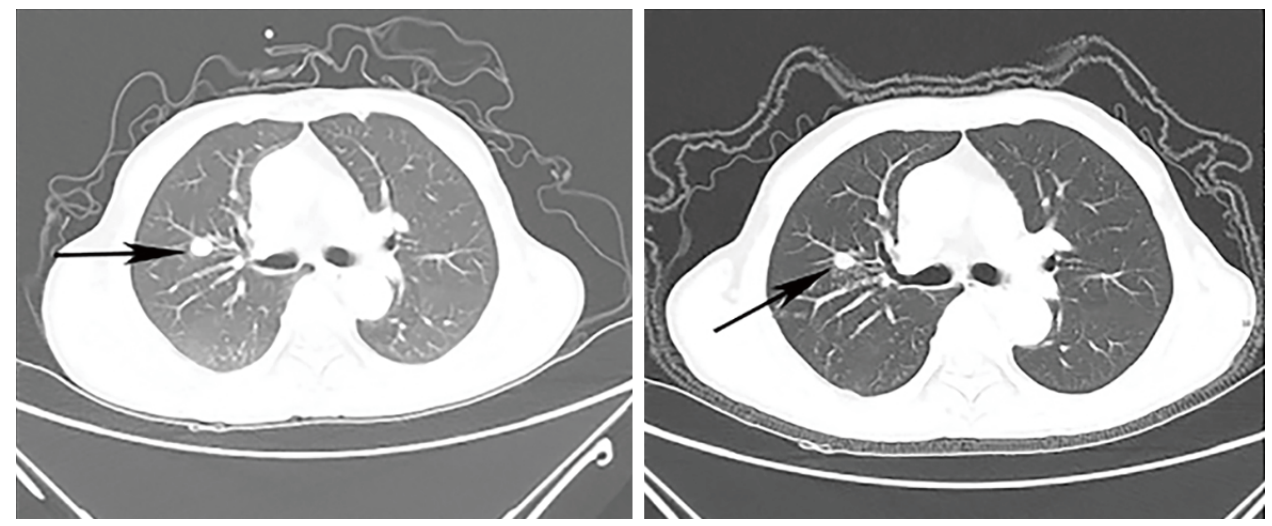

Figure $1 \mathrm{CT}$ scan of the chest demonstrates the reduction of the lung nodule size. The left was taken before RP and the size was $1.1 \mathrm{~cm} \times$ $1.2 \mathrm{~cm}$ (arrow). The right was taken after RP and ADT, shrinking to $0.8 \mathrm{~cm} \times 1.1 \mathrm{~cm}$ (arrow). RP, radical prostatectomy; ADT, androgen deprivation therapy.

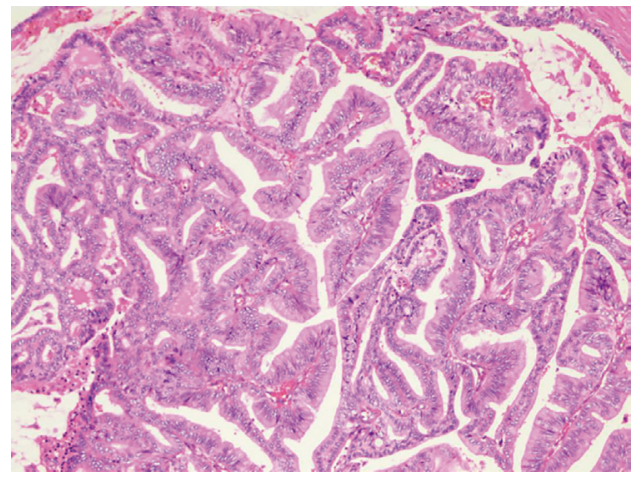

Figure 2 Prostate ductal adenocarcinoma type B, diffuse large acinar papillary tubular adenocarcinoma in the parenchyma of the prostate, resembling endometrial adenocarcinoma. HE staining, $\times 100$.

made this case difficult to be managed. We present the following article in accordance with the CARE reporting checklist (available at http://dx.doi.org/10.21037/apm20-386).

\section{Case presentation}

A-72-year-old male patient was admitted with complaints of frequent urination of 6 days duration. Neither hematuria nor fever were found during these days. The PSA value (prostate-specific antigen) was $12.64 \mathrm{ng} / \mathrm{mL}$, which was much higher than the normal standard. Prostatic MRI revealed a lump in the 3-6 o'clock position of the middle and $\mathrm{PZ}$ area of apex of prostate, PI-RADS 4-5 scores. However, there was no nodule can be touched by digital rectal examination except shallow median groove and

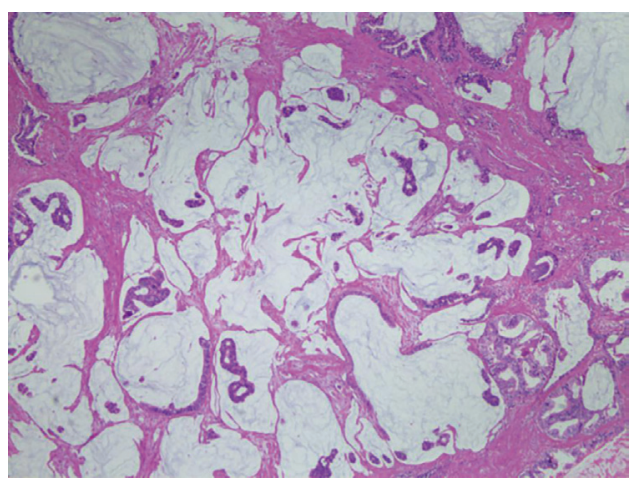

Figure 3 Prostate mucinous adenocarcinoma, interstitial mucus lake formation, floating adenoid tissue cancer cells. HE staining, $\times 100$.

uneven surface. To clarify the diagnosis, transperineal prostate biopsy guided by transrectal ultrasound was performed. The pathology revealed that the lump was acinar adenocarcinoma, and part of it was mucinous adenocarcinoma. The Gleason score was $4+3=7$. Wholebody bone scintigraphy and the chest CT scan showed that there was no bone metastasis but a lung nodule measuring $1.1 \mathrm{~cm} \times 1.2 \mathrm{~cm}$ (Figure 1, left). We then communicated with the patient to perform a biopsy to confirm whether this lung nodule was a metastasis, but the patient refused to take the risk of the hilum damage. Furthermore, He denied any family history of genetics or cancer and never smokes.

The patient was treated with RP very soon. Clinical pathology suggested that the mass with a diameter of $0.5-2 \mathrm{~cm}$ was acinar adenocarcinoma with focal ductal and mucinous adenocarcinoma differentiating (Figures 2,3; HE staining, $\times 100$ ). Gleason score was modified to $4+4=8$. 


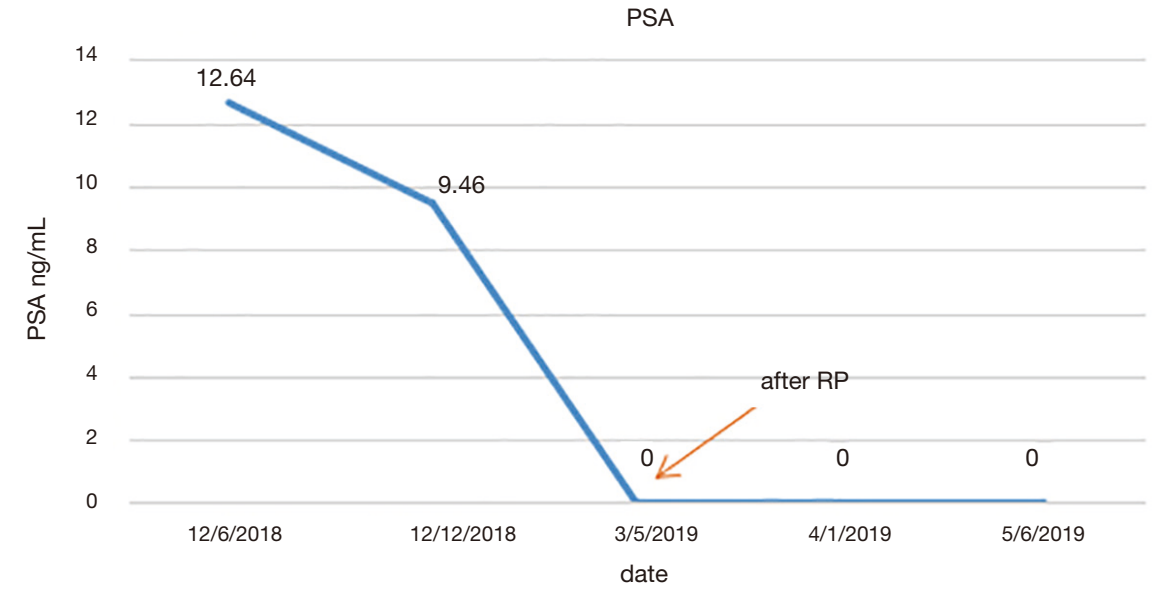

Figure 4 The PSA change chart. PSA, prostate-specific antigen.

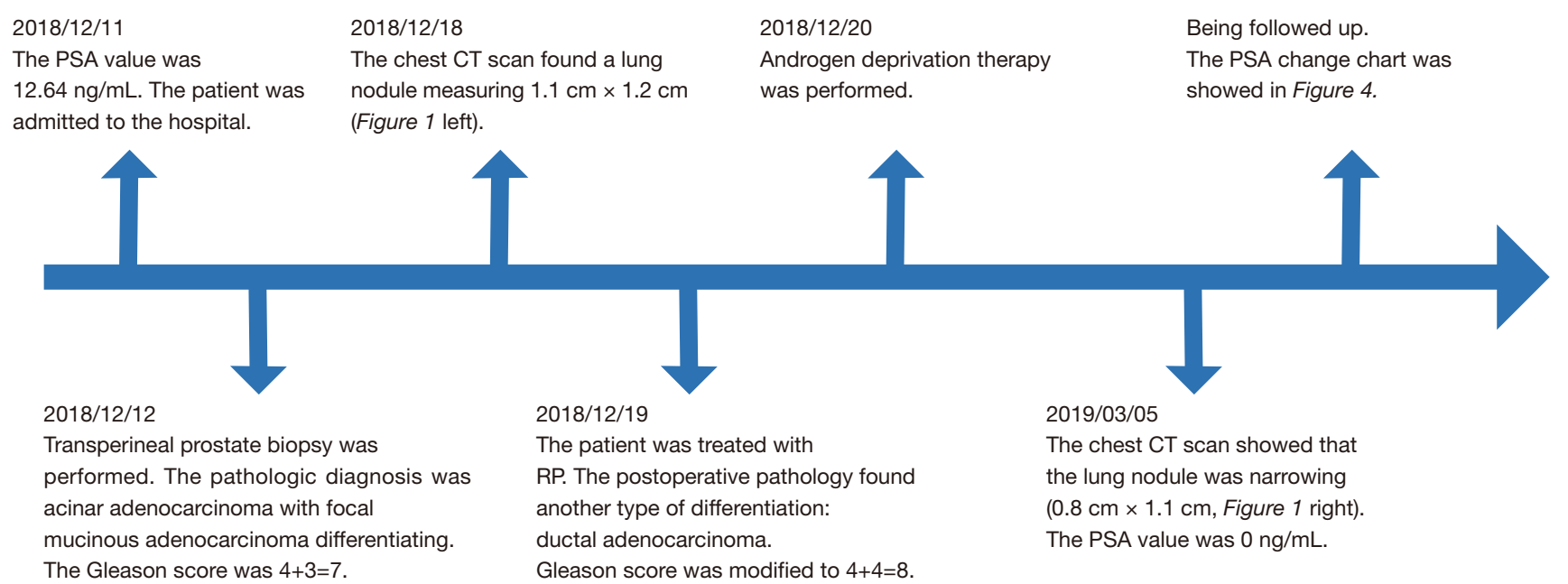

Figure 5 The timeline of the major diagnosis and treatment procedures.

The cancer tissue was located in the bilateral peripheral zone, with a wide and multifocal distribution. What's more, the cancer tissue showed a strong invasiveness involved prostatic capsule tissue, circumferentially cut edge, incision margin of bladder neck, right vas deferens and nerves. The final stage of the PCa was pT4N0M1c, stage IVB according to the TNM classification (AJCC 8th edition).

ADT (Goserelin acetate $3.6 \mathrm{mg}$ per 28 days) was performed immediately after operation and the patient recovered very well. He was asked to check the PSA every month and chest CT scan every 3 months. The patient showed good adherence. The PSA change was drawn in the blow (Figure 4) and the CT scan gave a good news that the lung nodule showed a shrinking trend which from $1.1 \mathrm{~cm} \times$ $1.2 \mathrm{~cm}$ to $0.8 \mathrm{~cm} \times 1.1 \mathrm{~cm}$ (Figure 1). He is now, 12 months later, no adverse or unanticipated events occurred, being followed up. The major diagnosis and treatment procedures of this patient were drawn as a timeline in Figure 5.

\section{Discussion}

The primary malignant tumor of prostate is acinar adenocarcinoma. DAP is a kind of papillary malignant tumor which mainly occurs in ductal and urethra of prostate. It is a rare subtype of PCa. The incidence of DAP alone is $0.4-0.8 \%(5,6)$, and most of DAP is combined with acinar adenocarcinoma which is account for $5 \%$ of $\mathrm{PCa}(7)$. 
Up to now, there is no guideline for the treatment, mainly using the treatment plan of prostate acinar adenocarcinoma with the same Gleason score, including RP, ADT, systemic chemotherapy and brachytherapy etc.

Of all PCa types, the incidence of mucinous adenocarcinoma is about $0.2 \%$ (8). Negative staining for PSA makes this primary subtype of prostate tumor difficult to diagnose in the early stage (9). By the time of diagnosis, patients usually have the symptoms of dysuria, lumbosacral pain and even lung or other metastases discomfort. Some studies have suggested that this subtype has a more aggressive behavior than typical acinar adenocarcinoma $(8,10)$, while others have shown these two types have a similar outcome (11). In addition, there is also a dispute over the therapy of mucinous adenocarcinomas. Lane et al. (11) found that there was no correlation between ADT and biochemical recurrence-free survival by studying 32 patients with mucinous adenocarcinoma or prostate adenocarcinoma with focal mucin. While Zhang et al. (9) considered that the patients, whose specimen stained positive for PSA, was responsive to $\mathrm{ADT}$, resulting in a better prognosis. However, they all agreed that RP should be the first choice which would make a good prognosis for patients. At the 2014 ISUP consensus conference (12), it reached a consensus that the grade of mucinous adenocarcinomas should be based upon the underlying architecture of the tumour, ignoring the mucinous component. This consensus made a great difference to grade mucinous adenocarcinomas and consequent treatment. In this case, considering multiple cancer metastases in the tissues submitted for pathological examination, high PSA level and lung metastasis, we took the ADT after RP to alleviate the metastases and improve the life quality of patient.

In particular, the lung nodule couldn't be ignored in this case. We all know that PCa most often metastasizes to bone and pelvic lymph nodes, except these all belong to atypical metastasis (13). In atypical metastasis, lung or pleura metastasis is especially rare.

Although some literatures reported that ADT had no significant effect on mucinous or ductal adenocarcinoma, we adopted this treatment actively to extend survival time. This is a successful case that RP united ADT to treat multidifferentiated PCa complicated by lung metastasis. The experience or guidelines for treating such patients has never been reported. After 12 months follow-up, there was no recurrence or metastasis, but the nature of the lung nodule is still unclear. Since the follow-up time is short yet, the prognosis needs longer follow-up to full understand the outcome of this rare patient. Without bearing the economic and physical burden from radiotherapy or chemotherapy, the patient's condition has been well controlled and his survival has been extended. He said he was very satisfied with the treatment.

In conclusion, this case shows that RP combined with ADT is helpful for advanced $\mathrm{PCa}$ with organ metastases and multiple differentiation. Further follow-up is warranted to confirm the curative effect.

\section{Acknowledgments}

Funding: The study was supported by the Six Talent Peak Project of High-level Talents in Jiangsu Province (WSW-017); 333 High-level Talents Training Project in Jiangsu Province, Professional from Six-Pronged TopTalent Program (LGY2018053); Qing Lan Project of Jiangsu University (JX2161015100); The Fifth Batch of Outstanding Young and Middle-aged Teachers Support Program of Nanjing Medical University; A Project Funded by the Priority Academic Program Development of Jiangsu Higher Education Institutions (JX10231802); Postgraduate Research \& Practice Innovation Program of Jiangsu Province (KYCX19_1159) and International Exchange and Cooperation Program for Postgraduates of Nanjing Medical University.

\section{Footnote}

Reporting Checklist: The authors have completed the CARE reporting checklist. Available at http://dx.doi.org/10.21037/ apm-20-386

Conflicts of Interest: All authors have completed the ICMJE uniform disclosure form (available at http://dx.doi. org/10.21037/apm-20-386). The authors have no conflicts of interest to declare.

Ethical Statement: The authors are accountable for all aspects of the work in ensuring that questions related to the accuracy or integrity of any part of the work are appropriately investigated and resolved. All procedures performed in studies involving human participants were in accordance with the ethical standards of the institutional and/or national research committee(s) and with the Helsinki Declaration (as revised in 2013). Written informed consent was obtained from the patient for publication of this manuscript and any accompanying images. 
Open Access Statement: This is an Open Access article distributed in accordance with the Creative Commons Attribution-NonCommercial-NoDerivs 4.0 International License (CC BY-NC-ND 4.0), which permits the noncommercial replication and distribution of the article with the strict proviso that no changes or edits are made and the original work is properly cited (including links to both the formal publication through the relevant DOI and the license). See: https://creativecommons.org/licenses/by-nc-nd/4.0/.

\section{References}

1. Ferlay J, Colombet M, Soerjomataram I, et al. Cancer incidence and mortality patterns in Europe: Estimates for 40 countries and 25 major cancers in 2018. Eur J Cancer 2018;103:356-87.

2. Osunkoya AO. Mucinous and secondary tumors of the prostate. Mod Pathol 2018;31:S80-S95.

3. Kan RW, Kan CF, Wong JH, et al. Ductal adenocarcinoma of the prostate: a Hong Kong case series. Int Urol Nephrol 2014;46:2133-7.

4. Cohen RJ, O'Brien BA, Wheeler TM.

Ductal adenocarcinoma of the prostate. Hum Pathol2011;42:605-6; author reply 606-7.

5. Bostwick DG, Kindrachuk RW, Rouse RV. Prostatic adenocarcinoma with endometrioid features. Clinical, pathologic, and ultrastructural findings. Am J Surg Pathol 1985;9:595-609.

6. Epstein JI, Woodruff JM. Adenocarcinoma of the

Cite this article as: Cui Y, Miao C, Xu A, Wang Z, Liu B. Acinar with ductal and mucinous adenocarcinoma of prostate cancer complicated with lung metastasis: a case report and literature review. Ann Palliat Med 2021;10(2):2366-2370. doi: 10.21037/apm-20-386 prostate with endometrioid features. A light microscopic and immunohistochemical study of ten cases. Cancer 1986;57:111-9.

7. Greene LF, Farrow GM, Ravits JM, et al. Prostatic adenocarcinoma of ductal origin. J Urol 1979;121:303-5.

8. Ro JY, Grignon DJ, Ayala AG, et al. Mucinous adenocarcinoma of the prostate: histochemical and immunohistochemical studies. Hum Pathol 1990;21:593-600.

9. Zhang L, Zhang L, Chen M, et al. Incidental discovery of mucinous adenocarcinoma of the prostate following transurethral resection of the prostate: A report of two cases and a literature review. Mol Clin Oncol 2018;9:432-6.

10. Epstein JI, Lieberman PH. Mucinous adenocarcinoma of the prostate gland. Am J Surg Pathol 1985;9:299-308.

11. Lane BR, Magi-Galluzzi C, Reuther AM, et al. Mucinous adenocarcinoma of the prostate does not confer poor prognosis. Urology 2006;68:825-30.

12. Epstein JI, Egevad L, Amin MB, et al. The 2014 International Society of Urological Pathology (ISUP) Consensus Conference on Gleason Grading of Prostatic Carcinoma: Definition of Grading Patterns and Proposal for a New Grading System. Am J Surg Pathol 2016;40:244-52.

13. Vinjamoori AH, Jagannathan JP, Shinagare AB, et al. Atypical metastases from prostate cancer: 10 -year experience at a single institution. AJR Am J Roentgenol 2012;199:367-72. 